\title{
Facial Expression Recognition from Visual Information using Curvelet Transform
}

\author{
Pratiksha Singh \\ Surabhi Group of Institution Bhopal
}

\author{
Nitesh Dodke \\ Surabhi Group of Institution Bhopal
}

\begin{abstract}
In the last decade, facial expression recognition has attracted more and more interest of researchers in the computer vision community. Facial expressions are a form of verbal communication, used to exchange social and emotional information in human-human-interaction. By detecting the expression of a human and reacting proactively, many applications could benefit from automatic facial expression recognition systems, e.g. human-computer-interfaces or security systems. Further applications for expression recognition lie in driver safety and social sciences. In order to use facial expression recognition systems in real-world situations, it is essential to recognize expressions not only from front face images, but also from images containing faces with pose variations.

In This work a new feature extraction technique has introduced from still images using PCA on curvelet domain which has been evaluated on a well-known databases. Curvelet Transform has better directional and edge representation abilities, inspired by these attractive attributes of curvelets, we decomposed images into its curvelet subbands and apply PCA (Principal Component Analysis) on the selected subbands in order to create a representative feature set.
\end{abstract}

\section{Keywords}

Facial Expression, Curvelet Transform, MATLAB

\section{INTRODUCTION}

Programmed outward appearance acknowledgment has numerous applications in territories, for example, human-PC cooperation (HCI), feeling investigation, indexing and recovery of picture and video databases intuitive video, picture understanding, and manufactured face movement [1]. As per [2], existing ways to deal with outward appearance investigation can be classified into methodologies in light of geometric and appearance. Geometric elements incorporate shapes and positions of face parts, for example, the sides of the mouth, commotion and eyebrows. Appearance-construct techniques depend in light of skin movement and surface changes (distortions of the skin, for example, wrinkles, swells, and wrinkles. Dynamic surface acknowledgment can be seen as a speculation of appearance based methodologies [2]. At the end of the day, notwithstanding the above exhibited approach dynamic composition based methodology can be respected for outward appearance examination. Outward appearances can be considered as a dynamic composition as a result of face muscles action is powerful. Along these lines, the appearance and movements of dynamic composition can be considered in two headings, it implies that data of spatial and fleeting areas is joined together. Saha et al. [3] were perceived outward appearances by mix

of curvelet change and neighborhood parallel examples. Additionally, they utilized curvelet entropy for characterizing facial expressions. Though, they just probed the still picture and performed on JAFFE databases and last picture of picture groupings of Cohn-Kanade databases. Juxiang et al. [4]

were utilized curvelet sub-groups (i.e., low recurrence, initially itemized layer with 4 and 8 headings, second nitty gritty layer with 4 and 8 bearings) as components for acknowledgment. They analyzed their methodology on CohnKanade and JAFFE databases. Aleksic and Katsaggelos [1] proposed facial activity parameters as components depicting outward appearances and utilized multistream HMMs for acknowledgment. The framework is perplexing, making it hard to perform continuously. Bartlett et al. [5], [6] is performed distinctive routines, for example, optical stream, unequivocal element estimation (i.e., length of wrinkles and level of educational), ICA, and the utilization of Gabor wavelets [2]. Yeasin et al. [7] were utilized the flat and vertical parts of the stream as elements. At the edge level, the k-closest neighbor (NN) principle was utilized to infer a trademark fleeting mark for each video grouping. At the arrangement level, discrete Hidden Markov Models (HMMs) were prepared to perceive the fleeting marks connected with each of the fundamental expressions. This methodology can't deal with enlightenment varieties, in any case. Cohen et al. [8] were presented a Tree Augmented-Naive Bayes classifier for acknowledgment. Whatever, they just probed an arrangement of five individuals, and the exactness was just around 75 percent [9].

Outward appearances is super class of feelings, it implies feelings goes under the classification of outward appearances.

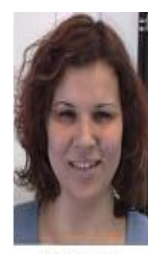

Happiness

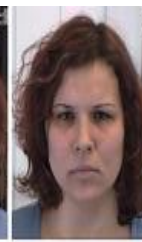

Sadness

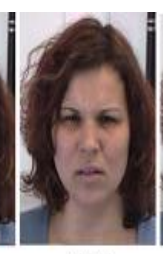

Anger

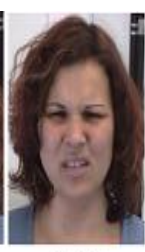

Disgust

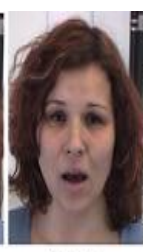

Surprise

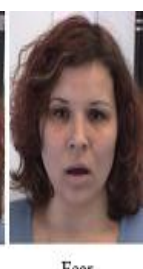

Fear
Figure: 1: Six Universal Emotions

Facial expression is broad class and emotion is the subclass of facial expression, because facial expression includes some cognitive process like thinking, boredom, drowsiness and so on, but Psychological researches have shown that only six central emotions are collectively coupled with different facial expressions: sadness, anger, surprise, fear, happiness, and disgust.

This thesis work has mainly addressed to recognition of these 6 central emotions but it can be customized to some added facial expression when needed. These six central emotions are shown in the figure 1.

\section{LITERATURE REVIEW}

Nakisa Abounasr et al. [1], This paper presents two new approaches for facial expression recognition based on digital curvelet transform and local binary patterns from three orthogonal planes (LBP-TOP) for both still image and image 
sequences. The features are extracted by using the digital curvelet transform on facial regions in still image. In this approach, some sub-bands correspond to angle of facial region is used. These sub-bands consist of more frequency information. The digital curvelet coefficients and LBPTOP are represented to combine spatio-temporal and spectral features for image sequences. The obtained results by our proposed approaches on the Cohn-Kanade facial expression database have acceptable recognition rates of $91.90 \%$ and $88.38 \%$ for still image and image sequences, respectively.

Sander Koelstra et al. [2], in this work, we propose a dynamic surface based way to deal with the acknowledgment of facial Action Units (AUs, nuclear facial signals) and their fleeting models (i.e., successions of worldly fragments: impartial, onset, pinnacle, and counterbalance) in close frontalperspective face recordings. Two ways to deal with demonstrating the elements and the appearance in the face locale of an info video are looked at: an expanded variant of Motion History Images and a novel strategy in light of Non inflexible Registration utilizing Free-Form Deformations (FFDs). The removed movement representation is utilized to infer movement introduction histogram descriptors in both the spatial and transient area. Per AU, a mix of discriminative, edge based Gentle Boost group learners and dynamic, generative Hidden Markov Models recognizes the vicinity of the AU being referred to and its transient fragments in an information picture succession. At the point when tried for acknowledgment of each of the 27 lower and upper face AUs, happening alone or in blend in 264 successions from the MMI outward appearance database, the proposed technique accomplished a normal occasion acknowledgment precision of 89.2 percent for the MHI system and 94.3 percent for the FFD strategy. The speculation execution of the FFD strategy has been tried utilizing the Cohn-Kanade database. At long last, we likewise investigated the execution on unconstrained expressions in the Sensitive Artificial Listener information set.

Ashirbani Saha et al. [3], this paper proposes the utilization of the blend of computerized curvelet change and nearby double examples for perceiving outward appearances from still pictures. The curvelet change is connected to the picture of a face at a particular scale and introduction. Nearby parallel examples are separated from the chose curvelet sub-groups to shape the spellbinding list of capabilities of the expressions. The normal of the components of a specific class of expression is considered as the agent highlight set of that class. The expression acknowledgment is performed utilizing a closest neighbor classifier with Chi-square as the disparity metric. Tests demonstrate that our technique yields acknowledgment rates of $93 \%$ and $90 \%$ in JAFFE and CohnKanade databases individually.

\section{APPROACH FOR FACIAL EXPRESSION RECOGNITION}

An FER (facial expression recognition) system has three components, namely Face-detection, Features extraction from face and Classification. Flow chart for proposed system is shown in the figure given below.

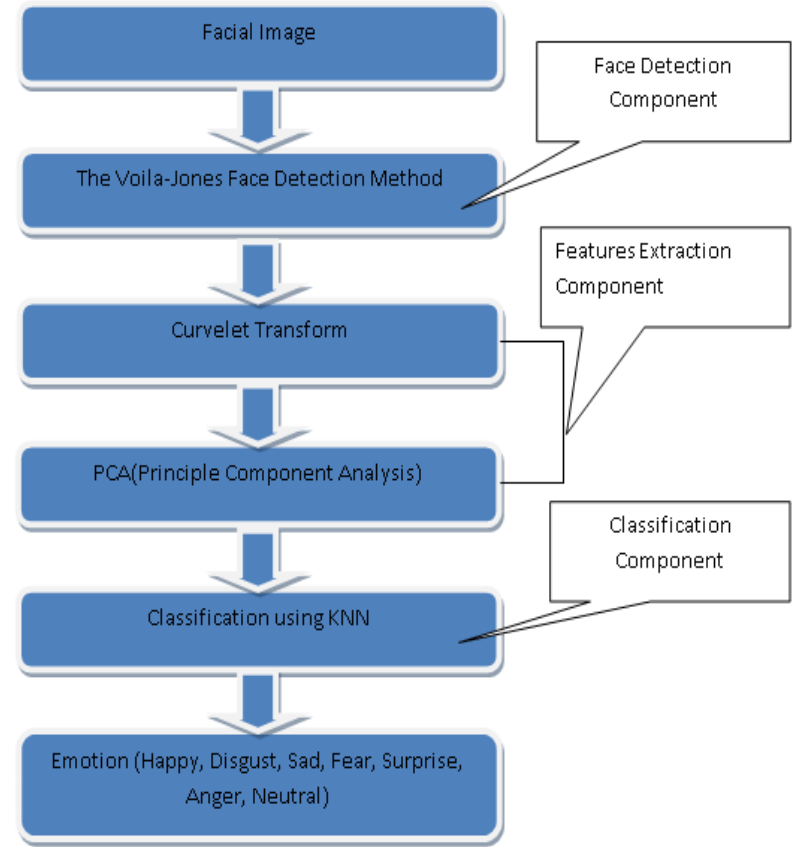

Figure 2: FER System

Face Detection: - This is the first and fundamental step of proposed facial expression recognition system. In this step, locate (identify) the face Viola region of the subject. For this purpose, Voila-Jones face detection method was used. -Jones method has successfully used for real time object identification in many computer visions and pattern recognition tasks. Drawback of this method is slow training, but advantages are very fast and accurate method for face detection [4]. Key feature of Voila-Jones face detection method is low false positive rate that is why we use this method in our proposed approach. Three major parts which contributes to Voila-Jones face detection [5]. First is "integral image" representation which allows features (used for detector) computation very fast. The second one is for selecting tiny set of features from very large set of features. For this very easy, accurate and precise classifier which is based on Ad boost learning algorithm is used. And third one is procedure for cascade combination of classifier, which spends more computation to assuring face like region while background region of the image discarded very fast. In the [15] authors revealed that this method has high detection rate so we use this in our system. In the figure below, shows the output of face detection on one image of JAFFE database.

\section{SIMULATION TOOLS}

In the below figure we show the starting screen of MATLAB. We use MATLAB because it is user friendly and it contains a number of libraries which is used in the work. 


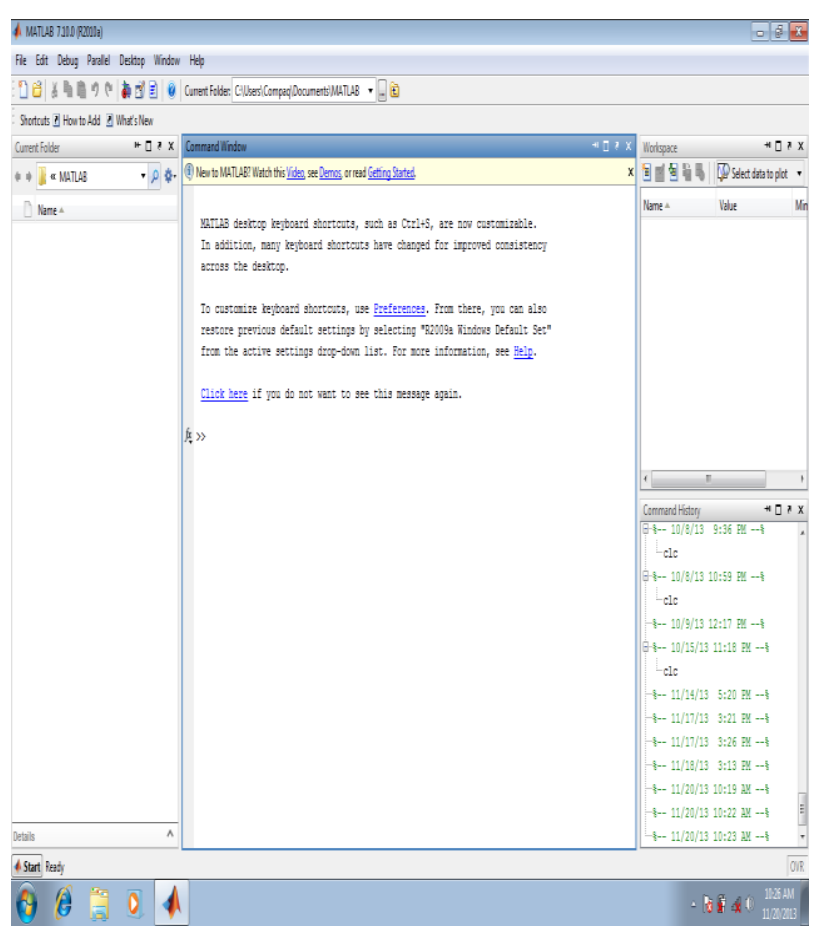

Figure 3: MATLAB Starting Window

This is the screen shot of our application; in this GUI we show the testing and training module.

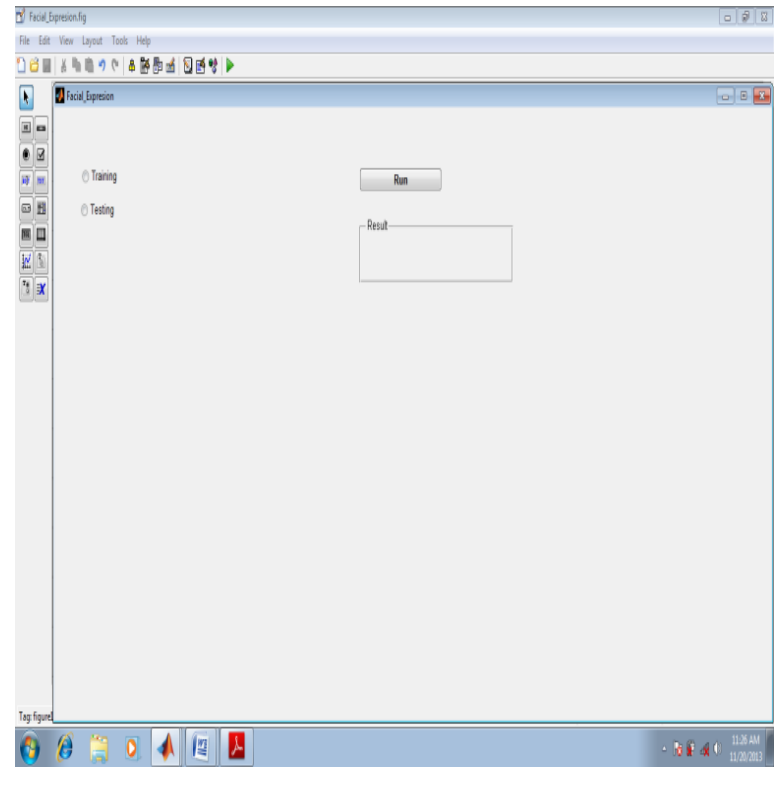

Figure 4: GUI of The Application

\section{SIMULATION}

In this section we expose and discuss the results of FER system which is based on proposed methodology for FER. We perform two experiments on proposed FER system with different number of training and testing images of JAFFE dataset. In first experiment out of 213 images, 143 images (on average of 2 images per expression per subject) for training purpose and rest 70 images (on average of 1 image per expression per subject) for testing purpose. Result of this experiment for 7 expressions is shown in the table below:
Table: 4.1 Result of Experiment FER System

\begin{tabular}{|c|c|c|c|c|c|c|}
\hline & $\begin{array}{c}\text { Ange } \\
\mathrm{r}\end{array}$ & $\begin{array}{c}\text { Disgus } \\
\mathrm{t}\end{array}$ & $\begin{array}{c}\text { Fea } \\
\mathrm{r}\end{array}$ & $\begin{array}{c}\text { Happ } \\
\mathrm{y}\end{array}$ & $\begin{array}{c}\text { Neutra } \\
\mathrm{l}\end{array}$ & $\begin{array}{c}\text { Sa } \\
\mathrm{d}\end{array}$ \\
\hline Anger & 10 & 0 & 0 & 0 & 0 & 0 \\
\hline Disgust & 0 & 10 & 0 & 0 & 0 & 0 \\
\hline Fear & 0 & 0 & 8 & 0 & 1 & 1 \\
\hline Happy & 0 & 0 & 0 & 8 & 1 & 1 \\
\hline Neutral & 0 & 0 & 0 & 0 & 10 & 0 \\
\hline Sad & 0 & 0 & 0 & 0 & 0 & 10 \\
\hline $\begin{array}{c}\text { Surpris } \\
\mathrm{e}\end{array}$ & 0 & 0 & 0 & 0 & 0 & 0 \\
\hline
\end{tabular}

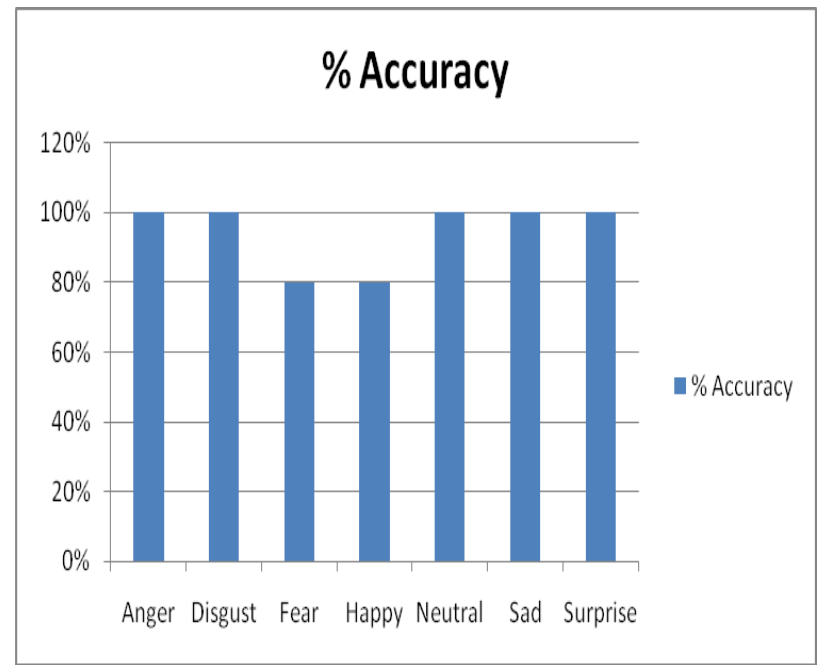

Figure 5: Accuracy of each expression for experiment

\section{CONCLUSION}

In this paper we have a couple of novel techniques for FER (Facial Expression Recognition) and emotion recognition from visual information. The key feature of proposed FER system is curvelet transform, which is used to extract features from facial images. Facial images contain curve edges of face which generally used to display an expression. For any FER system its vital need to extract curve edges information for expression recognition. Curvelet transform is well known multi-resolution transform which is used to extract curved edges information effectively from images.

\section{REFERENCES}

[1] B. Fasel and J. Luettin, "Automatic facial expression analysis: A survey," Pattern Recognition, vol. 36, pp. 259-275, 1999.

[2] C. Shan, S. Gong, and P.W. McOwan, "Facial expression recognition based on local binary patterns: A comprehensive study," Image and Vision Computing, vol. 27(4), pp. 803-816, 2008.

[3] Y. Tian, T. Kanade, and J. Cohn, Handbook of Face Recognition, chapter 11, Springer, 2005.

[4] M.S. Bartlett, G. Littlewort, M. Frank, C. Lainscsek, I. Fasel, and J. Movellan, "Recognizing facial expression: Machine learning and application to spotaneous behavior," in IEEE Computer Society Conference on Computer Vision and Pattern Recognition, 2005, vol. 2, pp. $568-573$.

[5] M.J. Lyons, J. Budynek, and S. Akamatsu, "Automatic classification of single facial images," IEEE 
Transactions on Pattern Analysis and Machine Intelligence, vol. 21(12), pp. 1357-1362, 1999.

[6] T. Mandal, A. Majumdar, and Q.M.J. Wu, "Face recognition by curvelet based feature extraction," in International Conference on Image Analysis and Recognition, LNCS, 2007, vol. 4633, pp. 806-817.

[7] A.A. Mohammed, R. Minhas, Q.M.J. Wu, and M.A. SidAhmed, "A novel technique for human face recognition using nonlinear curvelet feature subspace," in International Conference on Image Analysis and Recognition, LNCS, 2009, vol. 5627, pp. 512-521.

[8] T. Ojala, M. Pietikainen, and D. Harwood, "A comparative study of tex- ". ture measures with classification based on featured distribution," Pattern Recognition, vol. 29 (1), pp. 51-59, 1996.

[9] S. Liao, W. Fan, C.S. Chung, and D.Y. Yeung, "Facial expression recognition using advanced local binary patterns, tsallis entropies and global appearance features," in IEEE International Conference on Image Processing, 2006, pp. 665-668.

[10] M.J. Lyons, S. Akamatsu, M. Kamachi, and J. Goba, "Coding facial expressions with gabor wavelets," in IEEE International Conference on Automatic Face and Gesture Recognition, 1998, pp. 200-205. 